\title{
CUERPOS QUE IMPORTAN EN EL PATIO DE ATRÁS: PRECARIDAD Y HACER ESCUELA ESPECIAL EN CONTEXTOS DE POBREZA URBANA
}

\author{
BODIES THAT MATTER IN THE BACKYARD: PRECARITY AND SPECIAL \\ SCHOOL IN CONTEXTS OF URBAN POVERTY
}

\author{
Cintia Schwamberger ${ }^{1, *}$ \\ Silvia Grinberg ${ }^{2}$ (D)
}

\begin{abstract}
RESUMEN: El artículo propone, a través de resultados de investigación en la Región Metropolitana de Buenos Aires, caracterizar las tensiones de la inclusión-exclusión en una escuela de educación especial en contextos de pobreza urbana. A través de un trabajo etnográfico, describimos la precaridad que se vuelve doble en estas escuelas. La noción cuerpos en el patio de atrás problematiza las tensiones para fugar la exclusión. Proponemos que las lógicas de gestión de sí devienen formas de inclusión excluyente, donde docentes desarrollan micro-estrategias para el hacer escuela en la precariedad, los cuerpos importen.

Palabras clave: Educación especial. Discapacidad intelectual. Pobreza. Inclusión-exclusión escolar.
\end{abstract}

\begin{abstract}
This article proposes, through research results in the Metropolitan Region of Buenos Aires, to characterize inclusion-exclusion tensions in a special education school in urban poverty contexts. Through an ethnographic work, we describe the precarity that becomes double in these schools. The notion bodies in the backyard problematizes the tensions to escape exclusion. We propose that the logics of self-management become forms of exclusive inclusion, where teachers develop micro-strategies so that schooling becomes precarious, bodies matter.
\end{abstract}

Keywords: Special education. Intelectual disability. Poverty. School inclusion-exclusion.

1.Universidad Nacional de San Martín - Laboratorio de Ciencias Humanas -Buenos Aires, Argentina.

2.Universidad Nacional de San Martín - Laboratorio de Ciencias Humanas - Buenos Aires, Argentina.

*Autora correspondente: cintiaschwamberger@gmail.com

Número temático organizado por: Adriana da Silva Thoma (I.M.) e Betina Hillesheim

Financiado porConsejo Nacional de Investigaciones Científicas y Técnicas (CONICET) en base a la Beca doctoral Temas Estratégicos 2016-2021 


\section{Introducción}

No falto nunca a la escuela, aunque llueva y me embarre cuando salgo de casa. Prefiero venir a la escuela porque no hay goteras, vivo en una casa llena de agujeros, cuando llueve me mojo menos cuando viajo para la escuela. Pero cuando entro no me mojo más.

(Estudiante, varón, 20 años. Registro de campo propio, 2017.)

El presente artículo propone, a través de resultados de investigación en la Región Metropolitana de Buenos Aires (RMBA), caracterizar las tensiones de la inclusión-exclusión en una escuela de educación especial en contextos de extrema pobreza urbana ${ }^{1}$. A través de resultados de investigación caracterizamos aquello que hemos denominado como escolaridad precaria del sur global, la cual se vuelve doblemente excluyente cuando se trata de escuelas ubicadas en contextos marcados por la desigualdad social. Proponemos que si las dinámicas de inclusión excluyente (VEIGA-NETO; LOPES, 2007, 2011; ROSATO; ANGELINO, 2009; DE LA VEGA, 2008) configuran gran parte del hacer escuela en esos espacios de la urbe (GRINBERG, 2013, 2015), estas, se exacerban cuando se combina con la discapacidad intelectual. En este sentido, Veiga-Neto y Lopes (2007) señalan que se trata de dinámicas que las instituciones intentan reponer, sin conseguir que los destinatarios de esas políticas continúen al margen de la exclusión. Así, la inclusión, ocurre muchas veces como práctica de maquillaje (FERRANTE, 2016) y se vuelve una experiencia contradictoria para incluir, sin contar con ninguna o escasas condiciones para hacerlo. En suma, las políticas denominadas inclusivas no sólo no logran revertir los procesos excluyentes, sino que tienden a profundizarlos.

Proponemos a modo de hipótesis la noción cuerpos que importan en el patio de atrás a los efectos de problematizar las tensiones que atraviesan las escuelas de educación especial para propiciar procesos de inclusión y revertir la exclusión que atraviesan. Las lógicas de la gestión de sí y el hazlo tú mismo (GRINBERG, 2008, 2015) se traducen en micro-estrategias que desarrollan los docentes para hacer escuela en una materialidad, cuyo signo es la precariedad (BUTLER, 2006). A través de relatos de estudiantes y docentes proponemos elementos para la comprensión de esa inclusión excluyente. Seguidamente, como parte de esa tensión nos encontramos con modos de ese hacer que produce un plus que, como señala la frase del estudiante del epígrafe, permite no mojarse más. Nos referimos a las tensiones del hacer escuela cuando la discapacidad intelectual se combina con la pobreza urbana que marca los cuerpos. Cuerpos precarios pero que, como lo discutimos más adelante, en la escuela importan. Recuperamos el trabajo en terreno desarrollado en una escuela de educación especial que aloja a niños y jóvenes con discapacidad intelectual entre 8 y 25 años de edad ${ }^{2}$. La escuela se encuentra emplazada en uno de los distritos de la RMBA con los índices más altos de pobreza y desigualdad social.

La tensión inclusión/exclusión que recorre el artículo permite problematizar los procesos de escolarización de los y las estudiantes en contextos de extrema precariedad. Esta mirada se vuelve clave, entre otros, desde finales del siglo XX, para garantizar el derecho a la educación. Desde las últimas tres décadas y, especialmente a partir de grupos de personas con discapacidad se reconoce y amplía ese derecho a su inclusión

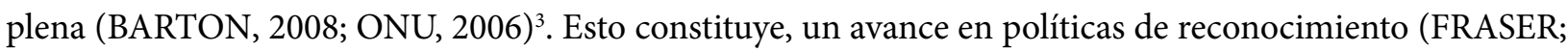
HONNETH, 2006), y un elemento fundamental para el impulso de procesos de inclusión (INFANTE; MATUS, 2009; LOPES, 2006, 2010). Los cambios se implementan de manera paulatina según cada institución en particular. Ello, debido al carácter natural que se atribuye al concepto moderno de inclusión, como un imperativo pero que se funde constantemente con la exclusión (VEIGA-NETO; LOPES, 2007, 2011). 
La noción cuerpos que importa en el patio de atrás, como categoría analítica del trabajo en terreno, permite ocuparnos de los modos en que docentes y estudiantes crean micro-estrategias para hacer frente a la precariedad (BUTLER, 2006). Se hace escuela con los recursos materiales, didácticos, herramientas de trabajo y el mobiliario que se consigue, en medio de dificultades edilicias o interrupción de servicios. Como refería un docente acá todo llega a cuenta gotas, en alusión a la implementación de políticas, proyectos y recursos que si llegan, lo hacen de manera inadecuada. Por otro lado, esa materialidad precaria se expresa en los cuerpos, en las sensaciones y en las expectativas de sujetos que, encuentran en la escuela un lugar para sobreponerse a la exclusión. Las micro-estrategias, que describiremos en el análisis, nos acercan a los modos en que consiguen hacer, parafraseando a Butler (2010), que cuerpos abyectos en la escuela importen. Asimismo, la autora señala que la modernidad produce vidas que se dirimen entre precariedad y precariedad, hacer escuela muchas veces se traduce en intervenir de forma tal que esas vidas abyectas encuentren maneras de plegarse hacia otros modos posibles.

En estos debates hemos organizado el artículo que en el primer apartado desarrollaremos la noción hacer escuela en tiempos de inclusión excluyente atendiendo a los procesos de escolarización de estudiantes con discapacidad en contextos de pobreza. Seguidamente, desde el enfoque etnográfico en educación proponemos indagar las formas que asume la vida escolar donde adquieren centralidad las micro-estrategias desarrolladas en la escolaridad y atraviesan los particulares del hacer escuela en el patio de atrás.

\section{Dinámicas de Inclusión/Exclusión en el Patio de Atrás}

Proponemos aquí, la noción cuerpos que importan en el patio de atrás con el objeto de problematizar las formas que asume la vida escolar atendiendo a las políticas tal como se inscriben en la cotidianeidad, y presentan modos particulares de hacer escuela (ROCKWELL, 2009; DAFUNCHIO; GRINBERG, 2013). En las escuelas donde desarrollamos distintos proyectos de investigación, asistimos a la producción de microestrategias derivadas de las políticas de responsabilización y empoderamiento, propio de tiempos gerenciales (GRINBERG, 2013, 2015). Nos referimos a situaciones donde los sujetos desarrollan estrategias y hacen frente a las demandas sociales, culturales y familiares que las atraviesan. Si esto es válido en general, en contextos de pobreza urbana, estas situaciones se exacerban porque como en el patio de atrás todo se acumula y los recursos si llegan, lo hacen a cuenta gotas.

El hacer cotidiano deviene hacer y hacer(se) en condiciones de precariedad (BUTLER, 2006) donde la excepción se vuelve norma. Hacer escuela acontece en un sinfín de situaciones donde se pasa a depender casi exclusivamente de las voluntades individuales y/o del voluntarismo (GRINBERG; BUSSI, 2013). Así las dinámicas para garantizar la inclusión devienen hacer-se cargo de la precariedad. Esto adquiere especial interés, desde los estudios de la gubernamentalidad (FOUCAULT, 2007; ROSE, 2007; VEIGA-NETO; LOPES,2007, 2011; GRINBERG, 2008, 2009), y, particularmente cuando se trata de docentes y estudiantes que asisten a escuelas especiales emplazadas en contextos de pobreza urbana. Ello, por medio de la (auto)organización y la (re) configuración de prácticas, consiguen desplegar y desarrollar micro-estrategias frente a una precariedad particular y un hacer(se) cargo de ella, para que la experiencia de los estudiantes importe. Debates actuales sobre precariedad (VEIGA-NETO; LOPES, 2011) reflexionan sobre los modos en que los sujetos arrojados a esa precariedad extrema, como formas de gubernamentalidad neoliberal, produce nuevas subjetividades y modos de resistencia, relacionadas con la agencia individual y la gestión de sí. Esto se vuelve clave en los procesos de escolarización que combinado con la discapacidad intelectual y la pobreza permite, visualizar nuevos modos de sujetos precarios.

Proponemos que la inclusión no puede pensarse separada de la exclusión, si bien, por un lado, tiende a eliminar las barreras y obstáculos, por el otro produce y profundiza las exclusiones que intenta revertir 
(VEIGA-NETO, 2005; FERRANTE, 2016), volviendo a la inclusión una gesta individual, una inclusión excluyente (VEIGA NIETO; LOPES,2007, 2011; ROSATO; ANGELINO, 2009). Así, retomando a Lunardi (2001) ya no se trata de pensar la inclusión como una cuestión binaria sino en cómo deconstruirla. Estudios críticos en discapacidad (MC RUER, 2006; YOUDELL, 2006; INFANTE et al., 2013; SARAIVA; LOPES, 2011; THOMA; HILLESHEIM, 2011) discuten los supuestos de la inclusión a partir del binomio normalidadanormalidad (FOUCAULT, 2006). Se apuesta a generar espacios críticos de reflexión para comprender las nociones de diferencia y diversidad ampliando, así, sus representaciones (INFANTE; MATUS, 2009).

Cuando se trata de escuelas donde la discapacidad intelectual se combina con la pobreza a esa vara de normalidad se suma la pregunta por los cuerpos abyectos (BUTLER, 2010). Mientras la inclusión reclama trabajar en esa vara incluso cuestionándola, las instituciones se enfrentan con la condición abyecta que pesa sobre la escuela y los alumnos (GRINBERG, 2010). Retomar a Butler involucra reflexionar la producción social de los cuerpos que importan y la normatividad que define sus términos. Aquello que no encaja en los discursos producidos por sus límites se convierte en un cuerpo siniestro, que acecha, irrumpe o amenaza. Butler (2010) propone una reformulación de la materialidad e invita a pensar los cuerpos como el efecto de dinámicas de poder. Es, en este sentido que se regulan los términos que habilitan la validez de los cuerpos, en donde unos importan más que otros. La pregunta por la inclusión nos arroja a otra mirada que, involucra la oportunidad de que otros cuerpos, sean capaces de ver en el cuerpo abyecto, la posibilidad de ser y habitar el mundo de un modo otro, un modo impropio, un modo impensado.

Entre esas tensiones cabe la pregunta por el hacer escuela donde docentes y alumnos encuentran en el cuerpo abyecto la posibilidad de un modo otro de ser, estar y habitar el mundo. Estas miradas se vuelven clave para entender algo de lo que ocurre a diario en las escuelas, donde llueve mucho en el camino, pero también se encuentra cobijo en su interior.

\section{La Escuela del Patio de Atrás: Breve Reflexión Metodológica}

Los relatos analizados son resultados de investigación en una escuela de educación especial de la RMBA entre 2016 y 2019. De este modo, nuestro objeto de estudio se conforma, atendiendo a los procesos de inclusión/exclusión de estudiantes con discapacidad intelectual, en la cotidianeidad de una escuela de educación especial emplazada en contexto de pobreza urbana. Para ello se realiza un trabajo desde un enfoque etnográfico post-estructuralista (YOUDEL, 2006; GRINBERG, 2013, 2015) a los efectos de comprender las dinámicas de dichos procesos (SINISI; MONTESINOS, 2009; VEIGA-NETO, 2005). Durante la estadía en la escuela, realizamos un trabajo de archivo, materiales históricos, normativas y propuestas pedagógicas a la vez que, un análisis de evolución de la matrícula. En una segunda instancia realizamos entrevistas flash y en profundidad a estudiantes (entre 15 y 20 años) y docentes mujeres de educación especial (de 40 y 50 años), combinándolo con observaciones participantes y no participantes (SCRIBANO, 2008) en espacios de recreación y clases ${ }^{4}$. Ello, en base a un criterio de selección específica, atendiendo al emplazamiento urbano de la matrícula escolar, las micro-estrategias desarrolladas y, el modo en que la hospitalidad y la pregunta por la enseñanza, en estos contextos, vuelve a los cuerpos con discapacidad intelectual, cuerpos que importan. Es este segundo cuerpo aquello que se discute en este texto. Se trata, de delinear prácticas y procesos de escolarización atendiendo a las condiciones y materialización de la precariedad en el hacer escuela (ROCKWELL, 2009) que, en esta escuela se conjuga en un sinfín de micro-estrategias para reponer los discursos de inclusión, alojar y permitir que todos los que ingresen a la escuela, estén allí y encuentren, algo del orden de volver posibles los deseos, tenga lugar.

La escuela especial se emplaza en uno de los distritos de la RMBA, con mayor densidad sociodemográfica asentada en el Conurbano Bonaerense ${ }^{5}$. Si bien, su edificio no está ubicado en ninguna 
de las villas miseria o slums $^{6}$ (GRINBERG; BUSSI, 2013) está próxima a todas. Por tratarse de la única escuela especial de gestión estatal para personas con discapacidad intelectual y dificultades en el desarrollo de la subjetividad, de doble jornada, con todos los niveles educativos, el servicio de inclusión y comedor, concurren muchos de los niños y jóvenes de esos barrios. La escuela está alejada del centro urbano cerca de una ruta nacional que atraviesa toda la región. La zona es un sector fabril. En las cuadras aledañas, el tránsito para llegar se configura con mucha circulación de autos, camiones, personas entrando y saliendo en sus horarios de trabajo, montacargas, paradas de colectivos y todo lo que dibuja una dinámica laboral fabril. Veredas muy angostas donde se camina pegado a la circulación de los autos que linda con un paredón de ladrillos y, desde la entrada de la escuela puede verse, como un muro que los separa. Cuando se camina por la calle al lado del muro, muchas veces se tiene la sensación de que hay algo que se quiere tapar, separar. Esta configuración del espacio urbano no es algo que los estudiantes pasen por alto, tal es así que, en un recreo, un grupo de estudiantes comenta "ni desde la terraza (el patio de arriba de la escuela) puedo ver lo que hay atrás de ese paredón, creo que es una fábrica, sólo veo camiones y gente entrando y saliendo, pero no se ve nada, no puedo ver qué hay" (estudiante, mujer 15 años, registro de campo propio, año 2016). En días de lluvia, por arreglos de las calles, su transitar complica el ingreso y egreso a la escuela. Algunos nos comentaban que no se ensuciaban cuando salían del barrio, pero sabían que se iban a embarrar cuando llegaran a la escuela. Tal como lo refiere un estudiante: "al final en mi casa sacaron la calle de tierra y ahora que no me embarro más, llego igual todo mugriento a la escuela” (varón, 18 años, registro de campo propio, 2016).

Cuando se termina la cuadra que separa a la escuela de la ruta, en la esquina se observa la entrada que, ocupa media manzana y linda con el paredón. Para ingresar hay que atravesar tres puertas, una la separa de la vereda que permanece rota hace dos años. La segunda permite ingresar al hall y la tercera, una puerta con vidrios que permite entrar a la institución. La parte de atrás de la escuela, es compartida con una escuela primaria y un jardín municipal. La escuela especial se emplaza en la denominada manzana de las escuelas. El patio de atrás, se comunica con el patio de la primaria. En momentos de recreo los jóvenes salen a jugar a la pelota a ese patio que en oportunidades "la cuelgan" como ellos comentan. La pelota se pasa hacia el patio de la escuela común, los chicos de este otro lado comienzan a gritar: - ¡la pelota! La pelota, "vuelve" sin ningún comentario, ellos contestan: - igracias! Esta escena se repite en el recreo donde los jóvenes eligen jugar al fútbol y pelotear. Casi como un ritual cotidiano, "colgar" y "devolver" se vuelve parte de un modo de comunicación en el patio de atrás que no se ven, porque los separa una medianera, pero se escuchan y juegan.

\section{Hacer Escuela en el Patio de Atrás: Cuando los Cuerpos Importan}

A lo largo del trabajo de campo hemos presenciado propuestas llevadas a cabo por la escuela, que habilita espacios, encuentros y modos de incluir a todos los que llegan. Estas propuestas se plantean como micro-estrategias y formar de recibir y hospedar a esos cuerpos que importan que se encuentran en situaciones de extrema precariedad, tanto por la discapacidad como las condiciones de pobreza en la que viven. Una docente lo expresa del siguiente modo: "esta escuela acepta e integra a los que están en el fondo del fondo" (mujer, 35 años, registro de campo propio, 2016). Desde esta perspectiva, describimos la situación de discapacidad que viven los estudiantes, la tensión inclusión/exclusión que hacen a la escuela una escuela del patio de atrás pero como lo expresan los docentes es una escuela donde entran todos, los que están en la lona y no tienen nada: acá recibimos a todos y cada vez son más. Tenemos pibes en lista de espera, que siguen esperando que haya vacante. Muchos no están escolarizados. A veces me pregunto, ¿dónde se quedan si no vienen a la escuela?, ¿dónde están? (Docente, mujer, 46 años, registro de campo propio, 2017). 
Esta expresión, recibimos a todos se vuelve clave para comprender el entramado complejo del hacer escuela especial en estos contextos. Un hacer escuela atravesado por una precariedad que se traslada a la materialidad de los cuerpos. ¿Dónde están si no están acá?, se pregunta la docente incluso cuando se trata de una escuela, cuya matrícula asciende año tras año y, los grupos se vuelven cada vez más numerosos, sin contar con los recursos necesarios para ello. Pregunta que acongoja y cuestiona la posibilidad de hacer escuela para sujetos cuyos cuerpos están atravesados por los límites materiales de la discapacidad y la pobreza. Son esas preguntas las que tensionan y frente a ello los docentes (re)hacen micro-estrategias para que esos cuerpos estén y permanezcan en la escuela. De hecho, esa pregunta adquiere un matiz particular cuando uno se encuentra con el detalle de la matrícula escolar. Al respecto, en la entrevista sobre el emplazamiento de los estudiantes, la docente nos comenta lo siguiente:

Yo te digo de dónde provienen, pero primero te cuento cómo me di cuenta sin tener que preguntar cuáles eran las zonas más pobres del partido. Me mudé hace poco a la zona, no la conocía, pero a partir de conocer los lugares donde vivían y viven los estudiantes pude ir dándome cuenta que estos lugares de donde provenían eran las zonas más pobres del partido. Todo eso sin conocer el lugar, los pibes te dicen de que barrio viene y te vas armando en tu cabeza, día tras día como es un día en su vida cotidiana, cómo viven, con quién viven, si comen, no comen, etc. En fin, acá vienen los más pobres de barrios precarios, de asentamientos, de las villas de la zona (entrevista docente, mujer, 50 años, 2016).

La materialidad de esos cuerpos, cuerpos marcados por la precariedad permite a esta docente entrever o suponer de dónde provienen sus estudiantes sin que ello implique ningún prejuicio trillado. La docente, convive con esa materialidad que atraviesa a los sujetos con los que trabaja y busca incansablemente estrategias para que el encuentro, se vuelva parte de una experiencia que potencie y posicione al sujeto, marcado por la exclusión, en un lugar de posibilidad. Ello, quizá porque como refiere Butler (2006), el ser precario es un ser atravesado por lo otro, por lo diferente y no puedo eliminar su presencia, sino que en ese encuentro cotidiano el otro se aparece como rasgo de mi propia corporeidad, de mi propia vida y me interpela como tal.

Hacer escuela especial en contextos de pobreza urbana en el siglo XXI, sin duda no es tarea fácil. La pregunta por la escolaridad atravesada por la discapacidad y la pobreza urbana adquiere especial interés cuando la lógica gerencial deja a sujetos e instituciones teniendo que desarrollar, micro-estrategias para mantenerse en pie (GRINBERG, 2008, 2013, 2015). La cotidianeidad de ese hacer viene a reponer y sostener de forma individualizada, en el caso por caso, los procesos de inclusión de los estudiantes y eso hace que la escuela sea muchas cosas, todas a la vez. Como lo señala la supervisora de la modalidad:

Es precisamente de lo que tiene que hacerse cargo la escuela en la realidad compleja que vivimos, hoy los chicos vienen no sólo a estudiar, vienen a comer, vienen a buscar un espacio donde encontrarse con sus pares, vienen a trabajar, a conseguir una pasantía o un puesto laboral. Todo esto hace a la escuela especial y mucho más (supervisora, mujer, 49 años, registro de campo propio, 2016).

Hacerse cargo es quizá la clave del hacer escuela en tiempos gerenciales. Tal es así que, en el cotidiano, observamos docentes levantando paredes, pintando la escuela, acompañando a las familias a hacer trámites para acceder a distintos beneficios, pidiendo turnos para el Certificado Único de Discapacidad (CUD), orientando a familias en cuestión de derechos, entre tantos otros: 
Recién vengo de desarrollo social, fui a averiguar para sacar el DNI a Juan, que no tiene familia. El tema es que yo soy ni su familiar, ni su tutora, está acá por orden judicial, pero no tiene documento, ni partida. Voy a ver qué podemos hacer (trabajadora social, mujer, 40 años, registro de campo propio, 2016).

La superposición de actividades como micro-estrategias resulta en responsabilidades atravesadas por la materialidad de las historias de la escuela y tensiona ese hacer en estos espacios urbanos (GRINBERG, 2010), que modulan los procesos de escolarización entre la inclusión-exclusión constantemente. El hacer(se) cargo de la precariedad es la modalidad propia del hacer en el fondo que no deja de expresarse como contracara de una experiencia en los docentes, muchas veces, de desasosiego, de sentir que ya no pueden más:

Acá hacemos lo que podemos, todos los días algo nuevo pasa. Recién vengo de buscar las computadoras para llevarlas a Biblioteca porque con la tormenta de ayer, saltó la térmica. Está todo en corto en esa sala y no se puede usar. Acá hace cortocircuito todo en cualquier momento, no sólo la electricidad (entrevista, docente, mujer, 41 años, 2016).

La escuela en cortocircuito como se menciona es parte de la trama escolar que viven a diario. Ello, los deja expuestos, viviendo en ese estar al límite que, sin duda, modula los procesos de escolarización. Así, en relación, con las tormentas una docente nos comenta un modo de llevar adelante el aprendizaje:

Estás dando clase y de repente empieza a llover, ya sabés que en cualquier momento te quedás a oscuras con los pibes. Pibes que muchas veces viven estas situaciones en sus hogares y otros que por h o por b no les gusta la oscuridad, o porque se ponen muy nerviosos cuando algo extraño sucede en la escuela. ¿Cómo enseñás matemática, prácticas del lenguaje, en esta situación? Así no se puede, algunas le ponemos garra y onda, pero a veces esta situación te desgasta y te saca todas las ganas. Pero seguimos por los pibes (docente, mujer, 48 años, registros de campo propio, 2016).

Es la tensión, la alta tensión, que hace saltar la térmica, y aparece la pregunta de cómo enseñar que atraviesa los modos de hacer escuela, en ese límite, que desdibuja responsabilidades. Entrar a la escuela, trabajar cotidianamente supone volverse experto en la gestión de lo intempestivo. Mientras la térmica no salte se transita el hacer escuela. Ello, hasta que lo intempestivo gana terreno y hay que bajar la térmica, re-setear y volver a empezar. Reponer los discursos de la inclusión implica que, año tras año, los docentes renueven energías y se carguen de electricidad, de la que no salta, para materializar micro-estrategias y nuevos proyectos. Se trata, en definitiva, de que la escuela sea una escuela dónde entren todos, de volverla hospitalaria, disponible. De hecho, muchas veces, lo consiguen como decía un alumno que a la escuela no falta nunca porque incluso se moja más en su casa que, caminando para llegar a ella.

Es en esta escena que la tensión inclusión-exclusión adquiere otras notas que importan retomar aquí, a modo de corolario. Allí en situaciones de precariedad y precariedad (BUTLER, 2006) que expuestos estudiantes y docentes, la escuela consigue habilitar una hospitalidad entre los detalles y micro-detalles que hacen al devenir de la vida escolar. Son, probablemente, esos instantes aquellos donde el hacer escuela en la precariedad produce un plus, donde la escuela deviene lugar para asumir una voz en los asuntos públicos, en los asuntos de la escuela, de las casas, de sus vidas. Un devenir otro, donde la vida abyecta acaece palabra otra en la que los cuerpos importan. 


\section{A Modo de Cierre: los Cuerpos que Importan en el Patio de Atrás}

A lo largo de este trabajo intentamos ofrecer herramientas para problematizar las tensiones de la inclusión-exclusión tal como son experienciadas en esta escuela de educación especial emplazada en contexto de pobreza urbana, donde docentes y estudiantes logran hacer(se) escuela en condiciones de precariedad. Una precariedad material de las instituciones y, de los cuerpos. Precariedad que ya no tiene que ver con una posición periférica, sino que atraviesa a la realidad en su conjunto y deviene norma. Los docentes reponen a diario los discursos de la inclusión por medio de micro-estrategias para que los estudiantes encuentren un espacio estable, contenedor, donde reciban cobijo, porque si no, como se pregunta la docente, ¿dónde están?

La inclusión se vuelve un imperativo, que incluye a todos porque, no podemos decir que no. Esto permite analizar las posibilidades de la escuela especial, a hacer(se) en el patio de atrás con lo que tienen, lo que pueden. Se encomienda a las escuelas y docentes responder a los principios de inclusión, mientras salta la térmica. Son los docentes llamados a resolver esta tensión entre la inclusión/exclusión con limitado o nulo acompañamiento. Es a través de esta institución que llegan y se implementan programas y beneficios sociales. Es en la escuela también dónde se hacen colectas para familias connecesidades y emergencias. Es en la escuela donde se entregan donaciones y materiales que provienen de distintos organismos e instituciones, para sostener la escolaridad. Es en la escuela dónde los estudiantes reciben vianda de comida para llevarse a sus casas y compartir con sus familiares. Es entre estas intensidades, que hacer escuela y docencia en el fondo se vuelve parte de un vaivén cotidiano que permea los límites del hacer, o más bien, en palabras de la supervisora, donde hacer deviene hacerse cargo.

El patio de atrás no es un lugar donde solo se confirma la imposibilidad. El patio de atrás habilita la comunicación, como un juego, entre muros. Incluso a veces queda la pregunta de si se trata de jugar al fútbol, o de generar ese momento donde al grito de ipelota por favor! vuelve el gracias. Es también tocarse con aquellos cuerpos abyectos que encuentran modos contingentes de estar, permanecer y atravesar el espacio-tiempo escolar. Es la escuela, tal como lo expresan los estudiantes, un lugar de valoración individual y colectivo porque es un espacio que los recibe, escucha y enseña. Es en ese momento donde hacerse cargo, se vuelve un hacer que a la vez habilite, quizá como un destello, un devenir-otro.

\section{Contribución de las Autoras}

Conceptualização: Schwamberger C and Grinberg S; Metodologia: Schwamberger C and Grinberg S; Redação - Primeira versão: Schwamberger C and Grinberg S.

\section{Notas}

1. Este proyecto se enmarca en la Beca Doctoral otorgada por el Consejo Nacional de Investigaciones Científicas y Tecnológicas (CONICET).

2. La escuela se encarga de estudiantes con discapacidad intelectual derivadas en su mayoría de dificultades madurativas leves del desarrollo; problemas de aprendizaje escolar; déficit atencional y trastornos emocionales de la subjetividad (TES). Asisten 499 estudiantes distribuidos en los dos servicios brindados. Por un lado, el servicio de inclusión, con 256 estudiantes incluidos en las escuelas regulares o comunes y, por el otro, el servicio de sede con 243 estudiantes. 
3. La Convención Internacional sobre los derechos de las Personas con Discapacidad (ONU,2006) marcó un hito como punto de partida y de llegada ante las luchas por el reconocimiento de Grupos de las Personas con Discapacidad. Por acople a ello, la Ley de Educación Nacional n. 26.206 establece el principio de inclusión educativa. Su ratificación a partir del 2008, Ley n. 26.378, acompañada por resoluciones ajustadas a las propias "necesidades y prioridades" de cada espacio del territorio. Por esto, en cada espacio del país, sostiene semejanzas y diferencias en función de la aplicación de las normativas y resoluciones enviadas desde los Ministerios de Educación.

4. Los estudiantes y docentes que forman parte de la muestra de la investigación, por medio del consentimiento informado han aceptado ser parte de ella para la utilización de sus voces en este artículo.

5. Denominación que refiere a la conformación de Partidos del Gran Buenos Aires, como un grupo integrado por 24 municipios que rodean a la ciudad de Buenos Aires.

6. En Argentina, se denomina villas a espacios urbanos densamente poblados en los cuales la mayor parte de su población -si no toda- vive en condiciones de pobreza; fruto del empobrecimiento de amplios sectores sociales. Hoy en día, la provisorio dejó paso a la permanente -de las viviendas, pero, lo que es más importante, de su precariedad-: a las familias que viven allí desde hace muchos años, se agregan, cada día, y aún más con cada crisis socio-económica, más familias que, aunque posiblemente tengan planes de irse, saben que lo más probable es que tengan que quedarse (GRINBERG; BUSSI, 2013).

\section{REFERENCIAS}

BARTON, L. Políticas sociales y discapacidad. Madrid: Morata, 2008.

BUTLER, J. Vida precaria. El poder del duelo y la Violencia. Buenos Aires: Paidós, 2006.

BUTLER, J. Cuerpos que importan. Sobre los límites materiales y discursivos del "sexo". Buenos Aires: Paidós, 2010.

DAFUNCHIO, S.; GRINBERG, S. Biopolítica y experiencia de la escolaridad en contextos de extrema pobreza urbana y degradación ambiental. Magistro, Buenos Aires, v. 7, n. 14, p. 245-269, 2013. Disponible en: https://ri.conicet.gov.ar/bitstream/handle/11336/35442/CONICET_Digital_Nro.60222a 29-e690-4135a705-cd6ec929b3b5_D.pdf? sequence=5\&isAllowed=y. Acceso: 4 oct. 2014.

DE LA VEGA, E. Las trampas de la escuela integradora. Buenos Aires: Novedades Educativas, 2008.

FERRANTE, C. Las otras caras de la moneda: "discapacidad" y limosna en el norte de Chile. In: VERGARA, G.; DE SENA, A. (comps.). Geometrías Sociales. Buenos Aires: ESE Editora, 2016. p. 273-288.

FOUCAULT, M. Los Anormales. Buenos Aires: Fondo de Cultura Económica, 2006.

FOUCAULT, M. Nacimiento de la biopolítica. Curso en el Cóllege de France: 1978-1979. Buenos Aires: Fondo de Cultura Económica, 2007.

FRASER, N.; HONNETH, A. ¿Redistribución o reconocimiento? Madrid: Morata, 2006.

GRINBERG, S. Educación y poder en el siglo XXI. Gubernamentalidad y pedagogía en las sociedades de gerenciamiento. Buenos Aires: Miño y Dávila, 2008. 
GRINBERG, S. Tecnologías del gobierno de sí en la era del gerenciamiento: la autoayuda entre el narcicismo

y la abyección. Psicoperspectivas, Chile, v. VIII, n. 2, p. 293-308, 2009. Disponible en: http://www. psicoperspectivas.cl/index.php/psicoperspectivas/article/view/84/81. Acceso: 12 oct. 2017.

GRINBERG, S. Everyday banality in a documentary by teenage women: between the trivial and the extreme. schooling and desiring in contexts of extreme urban poverty. Gender \& Education, London, v. 22, p. 663663, 2010. https://doi.org/10.1080/09540253.2010.519601

GRINBERG, S. La comunidad fragmentada: gubernamentalidad y empoderamiento en territorios urbanos hiperdegradados. Revista Espacios, Rio Gallegos, v. 7, p. 154-172, 2013. https://doi. org/10.1590/0104-4060.53863

GRINBERG, S. De la disciplina al gerenciamiento, del examen al monitoreo. Rase, Madrid, v. 8, n. 2, p. 156173, 2015. Disponible en: https://ojs.uv.es/index.php/RASE/article/view/8373/7966. Acceso en: 15 mar. 2016.

GRINBERG, S.; BUSSI, E. Notas sobre la vida escolar en territorios hiperdegradados, 2013.

INFANTE, M.et al. Narrando la vulnerabilidad escolar: performatividad, espacio y territorio. Literatura y Lingüística, Santiago de Chile, n. 27, p. 281-308, 2013. https://doi.org/10.29344/0717621X.27.61

INFANTE, M.; MATUS, C. Policies and practices of diversity: reimagining possibilities for new discourses. Disability \& Society, Abingdon, v. 4, n. 24, p. 437-445, 2009. https://doi.org/10.1080/09687590902879049

LOPES, M. C. O lado perverso da inclusão - a exclusão. In: FÁVERO, A.A. et al. (orgs.). Sobre filosofia e educação: racionalidade e tolerância. Passo Fundo: UPF Editora, 2006. p. 207-218. Disponible em: http:// biblioteca.idep.edu.co/libros/Gubernamentalidad_Web.pdf. Acceso: 15 ene. 2017.

LOPES, M. C. et al. Inclusão e biopolítica. Caderno IHU Ideias, São Leopoldo, año 8, n. 144, 2010. Disponible em: http://www.ihu.unisinos.br/images/stories/cadernos/ideias/144cadernosihuideias.pdf. Acceso: 14 ene. 2021.

LUNARDI, M. L. Inclusão/exclusão: duas faces da mesma moeda. Revista Educação Especial, Santa Maria, v. 2, n. 18, p.112, 2001. https://doi.org/10.5902/1984686X

MC RUER, R. Crip theory: cultural signs of queernes and disability. New York: New York University Press, 2006.

ONU [ORGANIZACIÓN DE LAS NACIONES UNIDAS]. Convención Internacional sobre los Derechos de las Personas con Discapacidad. New York: ONU, 2006.

ROSE, N. The Politics of Life Itself: Biomedicine, Power, and Subjectivity in the Twenty-First Century. New Jersey: Princeton University Press, 2007.

ROCKWELL, E. La experiencia etnográfica. Historia y cultura en los procesos educativos. Buenos Aires: Paidós, 2009. 
ROSATO, A.; ANGELINO, M. A. (coords.). Discapacidad e Ideología de la Normalidad. Desnaturalizar el déficit. Buenos Aires: Novedades Educativas, 2009.

SARAIVA, K.; LOPES, M. C. Educação, Inclusão e reclusão. Currículo sem Fronteiras, v.11, n.1, p.14-33, jan./jun.2011. Disponible en: http://www.curriculosemfronteiras.org/vol11isslarticles/saraiva-lopes.pdf. Acceso: 29 oct. 2017.

SCRIBANO, A. El proceso de investigación social cualitativo. Buenos Aires: Prometeo, 2008.

SINISI, L.; MONTESINOS, M. P. Entre la exclusión y el rescate. Un estudio antropológico en torno a la implementación de programas socioeducativos. Cuadernos de Antropología Social, n. 29, jul. 2009. Disponible en: http://www.scielo.org.ar/scielo.php?script=sci_arttext\&pid=S1850-275X2009000100003 Acceso: 10 mar. 2015.

THOMA, A. S.; HILlESHEIM, B. (orgs.). Políticas de inclusão: gerenciando riscos e governando as diferenças. Santa Cruz do Sul: EDUNISC, 2011. p. 7-15.

VEIGA-NETO, A. Quando a inclusão pode ser uma forma de exclusão. In: MACHADO, A. M. et al. (orgs.). Educação inclusiva: direitos humanos na escola. São Paulo: Casa do Psicólogo, 2005. p. 51-70.

VEIGA-NETO; A.; LOPES, M. C. Inclusão e governamentalidade. Educação \& Sociedade, Campinas, v. 28, n. 100, p. 947-964, out. 2007. https://doi.org/10.1590/S0101-73302007000300015

VEIGA-NETO; A.; LOPES, M. C. Inclusão, exclusão, in/exclusão. Verve, Revista Semestral Autogestionária do Nu-Sol, São Paulo, v. 20, p. 121-135, 2011. https://doi.org/10.23925/verve.v0i20.14886

YOUDELL, D. Impossible bodies, impossible selves: exclusions and student subjectivities. Holanda: Springer, 2006.

Recebido: 01 Out. 2019

Aceito: 10 Set. 2020

Editoras Associadas:

Daniela Dias dos Anjos e Ana Luiza Bustamante Smolka 